\title{
Iridoids with Genipin Stem Nucleus Inhibit Lipopolysaccharide-Induced Inflammation and Oxidative Stress by Blocking the NF-kB Pathway in Polycystic Ovary Syndrome
}

\author{
Tao Zuo a,c Minghui Zhu ${ }^{b}$ Wenming Xuc Zhixia Wanga Hang Song ${ }^{a}$ \\ aDepartment of Pharmaceutical and Biological Engineering, School of Chemical Engineering, \\ Sichuan University, Chengdu, Sichuan, ${ }^{\circ}$ Reproductive Medicine Center, the Second Affiliated Hospital \\ of Chengdu University of Traditional Chinese Medicine, Chengdu, Sichuan, cJoint Laboratory of \\ Reproductive Medicine, SCU-CUHK, West China Second University Hospital, Sichuan University, \\ Chengdu, Sichuan, China
}

\section{Key Words}

Iridoid $\bullet$ Inflammation $\bullet$ Oxidative stress $\bullet$ PCOS $・$ NF-KB

\begin{abstract}
Background/Aims: Polycystic ovary syndrome (PCOS) is a common endocrine disorder in women, and it is usually characterized by chronic inflammation, oxidative stress, and altered microRNA expression. The aim of this study is to investigate how the effects of iridoids with genipin stem nucleus inhibit PCOS complications. The interactions between iridoids were investigated, as well. Methods: The chronic inflammation cell model was induced using lipopolysaccharide (LPS) in the RAW 264.7 and KGN cell lines. Levels of mRNA and protein expression were quantified using real time-quantitative polymerase chain reaction (RT-qPCR) and western blot analysis, respectively. The target of the iridoids was identified using the drug affinity responsive target stability (DARTS) method. The ability to scavenge free radicals was evaluated using the DPPH radical scavenging method and the ultra oxygen anion $\left(\mathrm{O}_{2}^{-}\right)$radical scavenging method. Results: The cells recovered from the inflammatory conditions and showed significantly decreased levels of interleukins after treatment with iridoids. The iridoids were demonstrated to target NF- $\mathrm{kB}$, inhibit the phosphorylation and degradation of IкB, inhibit the nuclear entry of NF-KB , and inhibit the expression of inflammatory factors. Though only genipin showed an efficient ability to scavenge $\mathrm{O}_{2}{ }^{-}$, the iridoids, IKB inhibitor (BAY 11-7085), and NF-KB inhibitor (PDTC) could inhibit LPS-induced oxidative stress on the cells, indicating that the iridoids exert their anti-oxidant effects via the NF-KB pathway. The expression levels of microRNAs (miRNAs) were also altered by LPS, but the iridoids could scarcely rescue the abnormal condition. Conclusion: Chronic inflammation may be an important incentive for oxidative stress and abnormal microRNA expression in PCOS, and iridoids can protect patients from inflammatory damage by regulating the NF-KB pathway.

Professor Hang Song

and Professor Minghui Zhu

KARGER

Department of Pharmaceutical and Biological Engineering, School of Chemical Engineering, Sichuan University, No.24, South Section 1, Yihuan Road, Chengdu, 610065 (Chin) and Reproductive Medicine Center, the Second Affiliated Hospital of Chengdu University of Traditional Chinese Medicine, NO.17, Section 4, Renmin South Road, Chengdu, 610041 (China) E-Mail hangsong@vip.sina.com/zhumh00@sina.com
\end{abstract}




\section{Cellular Physiology Cell Physiol Biochem 2017;43:1855-1865

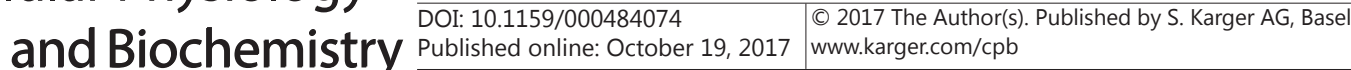

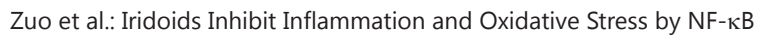

\section{Introduction}

Polycystic ovary syndrome (PCOS), one of the most common endocrine disorders in women of reproductive age, is often characterized by chronic inflammation, oxidative stress, and abnormal microRNA expression [1-4]. Since it is known that there is a close link between inflammation and oxidative stress, elevated oxidative stress usually results from and leads to an inflammatory condition [1]. It is difficult to separate inflammation from oxidative stress, and it has been thought in recent years that they should be regarded as a whole foundation of disease occurrence [5]. MicroRNAs (miRNA, miR) are endogenous, non-coding, singlestranded RNAs that are 20-24 nucleotides in length and modulate gene expression by binding to the 3' untranslated region (UTR) of target mRNA [6]. Many studies have shown altered miRNAs in serum, follicular fluid, adipose tissue, granulosa cell, and other tissues, and a portion of them were shown to play important roles in both PCOS and inflammation [7-9].

Eucommia Ulmoides is a unique Chinese traditional medicine that can be used in gynecology and obstetrics $[10,11]$. Iridoids are the primary components of eucommia, with genipin, geniposide, and geniposidic acid being the three major components of the iridoids [12]. Genipin has been shown to inhibit inflammation induced by various chemicals in vivo and in vitro and attenuate related pathological characteristics, such as liver injury [13-16]. Toll-like receptor (TLR), uncoupling protein-2 (UCP-2), cyclooxygenase 2 (COX-2), nuclear factor kappa $\mathrm{B}(\mathrm{NF}-\mathrm{\kappa} \mathrm{B})$ and many other factors were reported to be involved in the process of regulating genipin in inflammation [17-20]. Geniposide is also indicated to been efficient inhibitor of inflammation by regulating such proteins as TLR4, mitogen-activated protein kinase (MAPK), NF- $\kappa B$, and nuclear transcription factor activator protein 1 (AP-1) [21-28]. Thus, it has been considered that geniposidic acid also might have an anti-inflammatory effect, and the iridoids could be employed to treat inflammation in PCOS. Though many factors above are thought to be associated with the anti-inflammatory ability of the iridoids, no information regarding the specific target of the iridoids has been reported. The NF- $\kappa B$ signaling pathway is the core mechanism of the LPS-induced inflammatory process, and we focused on this process in this study to examine the anti-inflammatory mechanism of the iridoids and identify their target.

The iridoids seem to have the ability to alleviate oxidative stress in several conditions, as well, but to date, the immediate connections between the iridoids and oxidative stress have not been discovered $[29,30]$. In this study, free radical scavenging experiments were utilized to evaluate the anti-oxidant abilities of the iridoids, and the oxidative stress condition of the inflammatory process involved with LPS and the iridoids was also investigated. We aimed to determine the source of the potential anti-oxidant effects of the iridoids. Furthermore, the expression levels of several miRNAs were measured relative to PCOS in the above mentioned inflammatory process.

\section{Materials and Methods}

\section{Chemicals and reagents}

The iridoids (genipin, geniposide, and geniposidic acid, $\geq 98 \%$ ) were all purchased from Nanjing Jingzhu Bio-technology Co., Ltd. (Nanjing, China). Lipopolysaccharide (LPS) and dimethyl sulfoxide (DMSO) were obtained from Sigma (California, USA). Dulbecco's modified Eagle medium (DMEM), Dulbecco's modified Eagle medium: Nutrient Mixture F-12 (DMEM/F12), and trypsin were purchased from Gibico (Life, Invitrogen, California, USA). Fetal bovine serum (FBS) was purchased from Zhejiang Tianhang Biotechnology Co., Ltd. (Hangzhou, China). Trizol was purchased from Invitrogen (California, USA). Reverse transcription kit was purchased from TAKARA BIO Inc. and SYBR Select Master Mix was purchased from Life Technologies Corporation (Thermo Scientific, MA, USA). RIPA was purchased from Beyotime Institute of Biotechnology. Superoxide dismutase (SOD), catalase (CAT), glutathione peroxidase (GSH-Px), ultra oxygen anion scavenging $\left(\mathrm{O}_{2}^{-}\right)$, nitric oxide 


\section{Cellular Physiology Cell Physiol Biochem 2017;43:1855-1865 \\ \begin{tabular}{ll|l} 
DOI: 10.1159/000484074 & $\begin{array}{l}\text { O } 2017 \text { The Author(s). Published by S. Karger AG, Basel } \\
\text { www.karger.com/cpb }\end{array}$ \\
and Biochemistry Published onine: October 19, 201
\end{tabular}}

Zuo et al.: Iridoids Inhibit Inflammation and Oxidative Stress by NF- $\mathrm{kB}$

(NO) and malonaldehyde (MDA) assay kits were purchased from Nanjing Jiancheng Bioengineering Institute (Nanjing, China). BAY 11-7085 and PDTC were purchased from MCE (Shanghai, China) and Beyotime (Hunan, China), respectively. Other common organic reagents were all provided by Kelong Chemical Co., Ltd. (Chengdu, Sichuan).

\section{Cell culture}

RAW 264.7 cells were cultured in DMEM medium with 10\% FBS, and KGN cells were cultured in DMEM/F12 medium with $10 \%$ FBS. They were incubated at $37^{\circ} \mathrm{C}$ in a humidified atmosphere with $5 \% \mathrm{CO}_{2}$. Inflammatory conditions were induced by adding $2 \mu \mathrm{g} / \mathrm{mL}$ LPS to the RAW 264.7 and KGN cell lines. The iridoids (genipin, geniposide, and geniposidic acid) and the inhibitors (BAY 117085 and PDTC) were used at various concentrations as noted in the experiments.

\section{Reverse transcription-quantitative polymerase chain reaction (RT-qPCR)}

Total RNA was extracted using trizol according to the manufacturer's instructions, and total RNA concentration was measured using a Nanodrop 2000 (Thermo, MA, USA). The RNA was reverse transcribed using the kit in Thermal cycler (Bio-rad, California, USA), and the real-time qPCR was performed using the SYBR Green system in an ABI 7500 (Life Tech, Invitrogen, California, USA), strictly

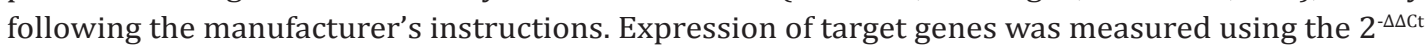
method, and the exogenous control gene was actin. Primers for microRNAs were purchased from Suzhou Ribo Life Science Co., Ltd. (Suzhou, China). The normal primers were designed using Primer Premier 5.0 (Premier, Canada) and synthesized by Chengdu Diqiao Biotechnology Co., Ltd. (Chengdu, China). The sequences of all primers used in this study are listed as followed: Mouse source: iNOS: forward: 5'-TCCTACACACCAAACTGTGTGC-3', reverse: 5'-CTCCAATCTCTGCCTATCCGTCTC-3'; IL-1ß: forward: 5'-GAGAATGACCTGTTCTTTGAAGTTGAC-3', reverse: 5'-TGAAGCTGGATGCTCTCATCAG-3'; IL-6: forward: 5'-TAGTCCTTCCTACCCCAATTTCC-3', reverse: 5'-TTGGTCCTTAGCCACTCCTTC-3'; IL-10: forward: 5'-GACAACATACTGCTAACCGACTCC-3', reverse: 5'-TTCACCTGCTCCACTGCCTTG-3'; Actin: forward: 5'-TTCCTTCTTGGGTATGGAAT-3', reverse: 5'-GAGCAATGATCTTGATCTTC-3'. Human source: iNOS: forward: 5'-GGAGCCAGCTCTGCATTATC-3', reverse: 5'-TTTTGTCTCCAAGGGACCAG-3'; IL-1 $\beta$ : forward: 5'-TTACAGTGGCAATGAGGATGAC-3', reverse: 5'-GTGGTGGTCGGAGATTCGTA-3'; IL6: forward: 5'-ACTCACCTCTTCAGAACGAATTG-3', reverse: 5'-CCATCTTTGGAAGGTTCAGGTTG-3'; IL8: forward: 5'-GAATGGGTTTGCTAGAATGTGATA-3', reverse: 5'-CAGACTAGGGTTGCCAGATTTAAC-3'; Actin: forward: 5'-GTTGTCGACGACGAGCG-3', reverse: 5'-GCACAGAGCCTCGCCTT-3'.

\section{Western blot}

To obtain the protein of cells, the cells were lysed using RIPA and centrifuged at $12,000 \times \mathrm{g}$ for 20 min to collect the supernatant. Protein concentration was measured using the BCA protein assay (Thermo Scientific, MA, USA). Equal amounts of protein were loaded onto an SDS-polyacrylamide gel, electrophoresed, and transferred to polyvinylidene difluoride membranes (Minipore, MA, USA). The membranes were blocked with $5 \%$ non-fat milk or bovine serum albumin (BSA) at room temperature for $1 \mathrm{~h}$ and incubated with primary antibodies at $4^{\circ} \mathrm{C}$ overnight. Each membrane was washed with TBST three times for $15 \mathrm{~min}$ followed by incubating with an HRP-conjugated secondary antibody (Zhongshan Jiangqiao, Beijing, China) at room temperature for $1 \mathrm{~h}$. Finally, each membrane was developed using an enhanced chemiluminescence (ECL) detection kit (Minipore, MA, USA) and visualized using X-OMAT BT film (Carestream, Toronto, Canada). The primary antibodies for TLR4, iNOS, IкB, NF-кB P65, Dicer1, tubulin, $\beta$-actin, and GAPDH were purchased from Proteintech Group (Chicago, USA), and the primary antibody for phosphorylated IкB was purchased from Abcam (Cambridge, UN).

\section{Immunofluorescence}

Cell slides for immunofluorescence were fixed using 4\% paraformaldehyde (PFA) at room temperature for 15 min and washed in PBS three times. Next, PBS with $0.2 \%$ Triton X-100 was used to permeabilize the cells at room temperature for $15 \mathrm{~min}$. The slides were blocked with $3 \%$ BSA at room temperature for $30 \mathrm{~min}$ and incubated with primary antibody at $4^{\circ} \mathrm{C}$ overnight. After that, the slides were washed three times with PBS, incubated with a secondary antibody (Zhongshan 
Jiangqiao, Beijing, China) at room temperature for $1 \mathrm{~h}$, and stained with DAPI for $10 \mathrm{~min}$. Finally, the slides were imaged using a confocal laser scanning microscope (Olympus, Japan) after being washed three times with PBS.

1. 1-diphenyl-2-picryl-hydrazyl (DPPH) and ultra oxygen anion $\left(\mathrm{O}_{2}^{-}\right)$free radical scavenging determinations, and SOD, CAT, GSH-PX, NO, and MDA measurements

DPPH ( $\geq 98 \%$ ) was purchased from TCI Development Co., Ltd. (Shanghai, China), and dissolved in ethanol at $0.05 \mathrm{mg} / \mathrm{mL}$. Appropriate iridoids were added to react with DPPH, and the solutions were measured at a wavelength of $519 \mathrm{~nm}$ using an ultraviolet spectrophotometer (Soptop, Beijing, China). Ultra oxygen anion free radical scavenging determination, SOD, CAT, GSH-Px, NO, and MDA measurements were measured strictly according to the manufacturer's instructions.

\section{Target identification}

The target of the iridoids was identified using drug affinity responsive target stability (DARTS) method, which takes advantage of a reduction in the protease susceptibility of the target protein upon drug binding [31]. In this study, cells were cultured with the iridoids for 30 min before incubating the extracted protein with thermolysin protease.

Statistical analysis

All data are presented as the means \pm SEMs and were compared by Student's t-test. Significance was set at $\mathrm{P}<0.05$.

\section{Results and Discussion}

Anti-inflammatory ability of the iridoids in PCOS

RAW 264.7 and KGN cells were incubated with either of control solution, LPS, and LPS plus the iridoid (genipin, geniposide, or geinposidic acid) for 24 hours. (Fig. 1) The results showed that the iridoids significantly inhibited the mRNA expression of IL-1 $\beta$, IL-6, IL-10and iNOS (Fig. 1A). LPS induction led to elevated protein expression of iNOS (Fig. 1B) in the RAW 264.7 cell line. Moreover, genipin inhibited LPS-induced over-secretion of nitrite in the medium (Fig. 1C). In addition, the iridoids also had an anti-inflammatory role in the KGN cell line (Fig. 1D, E).

Interleukins and iNOS are identified as common markers to evaluate the chronic inflammatory condition, and the iridoids showed efficient anti-inflammatory abilities by significantly inhibiting the expression of those markers. In this study, geniposidic acid, an iridoid with genipin stem nucleus, as well as genipin and geniposide, was demonstrated to be an efficient inhibitor of inflammation for the first time. The inflammatory process was induced in RAW 264.7 and KGN cell line (granulosa like tumor cell line), reflecting the immunological environment and the ovarian environment, respectively. An altered ovarian environment is an essential pathophysiological characteristic of PCOS, and granulosa cells are the dominant cell community in the ovarian environment, with roles in regulating the development of follicles and oocytes. Thus, determining the anti-inflammatory ability of the iridoids in the KGN cell line is important for the application of the iridoids in PCOS. However, it seemed that TLR4, the receptor of LPS, was not regulated by genipin and geniposide efficiently in either RAW 264.7 or KGN cells (for all online suppl. material, see www. karger.com/doi/10.1159/000484074, Suppl. Fig. 1A, B), and it did not play an important role when the iridoids inhibited inflammation. Nitrite levels were used to evaluate NO secretion, which is a common marker for inflammation. Genipin did not efficiently inhibit nitrite secretion in the study because of the potential apoptosis effect. In addition, neither geniposide nor geniposidic acid had effects on NO secretion. 


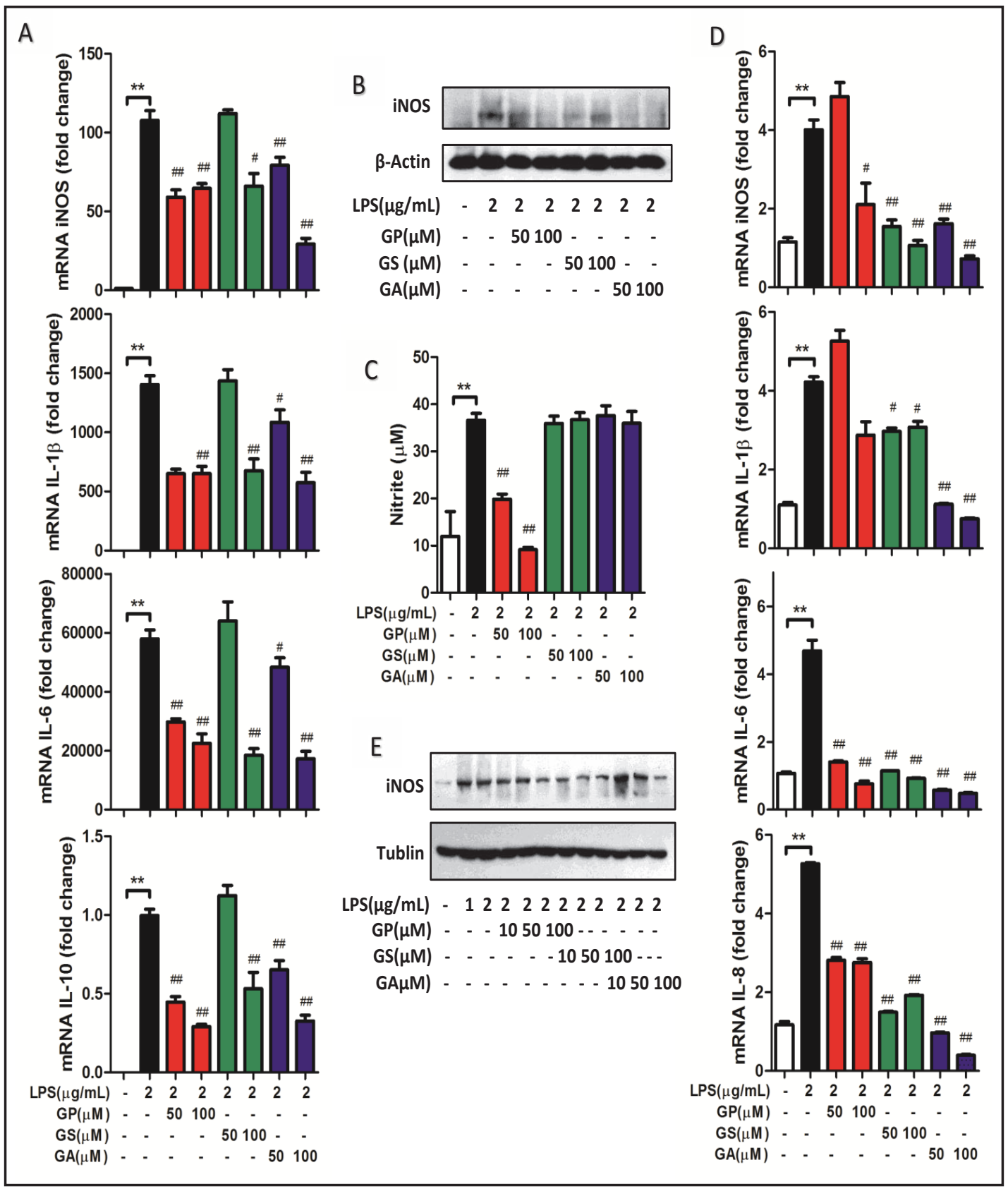

Fig. 1. RAW 264.7 and KGN cells were incubated with control solution, LPS, and LPS plus the iridoid (genipin (GP), geniposide (GS), or geniposidic acid (GA)) respectively for 24 hours. The results showed that the iridoids significantly inhibited the elevated mRNA expressionof IL-1 $\beta$, IL-6, IL-10 and iNOS (A), and the elevated protein expression of iNOS (B) induced by LPS in RAW 264.7 cell line. Moreover, genipin inhibited LPS-induced over-secretion of nitrite in the medium (C). In addition, the iridoids also played antiinflammatory role in KGN cell line (D, E). ${ }^{*} \mathrm{P}<0.0 .5$, ${ }^{* *} \mathrm{P}<0.01$; by contrast with LPS group, ${ }^{\#} \mathrm{P}<0.05$, ${ }^{\#} \mathrm{P}<0.01$.

\section{$N F-\kappa B$ signaling pathway in the inflammatory process}

RAW 264.7 cells were pre-incubated with control solution, genipin $(100 \mu \mathrm{M})$, geniposide $(100 \mu \mathrm{M})$, or geniposidic acid $(100 \mu \mathrm{M})$ for 2 hours before being incubated with LPS $(2 \mu \mathrm{g} / \mathrm{mL})$ for different times $(0,15,30$, and $60 \mathrm{~min})$. The results showed that all iridoids efficiently attenuated the LPS-induced elevated I $\mathrm{B}$ phosphorylation

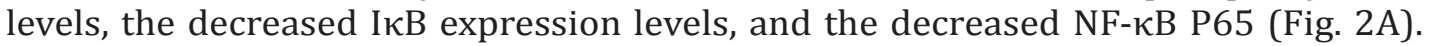


In addition, LPS-induced nuclear transfer of NF- $\kappa$ B P65 from the cytoplasm in RAW 264.7 was also blocked by the iridoids (Fig. 2B). RAW 264.7 cells were treated with iridoids of various concentrations for 30 minutes, and the lysates were subjected to thermolysin digestion. Though thermolysin efficiently degraded all the proteins (Fig. 2C), pretreatment with genipin or geniposide could block the degradation of NF- $\mathrm{KB}$ P65 (Fig. 2D).

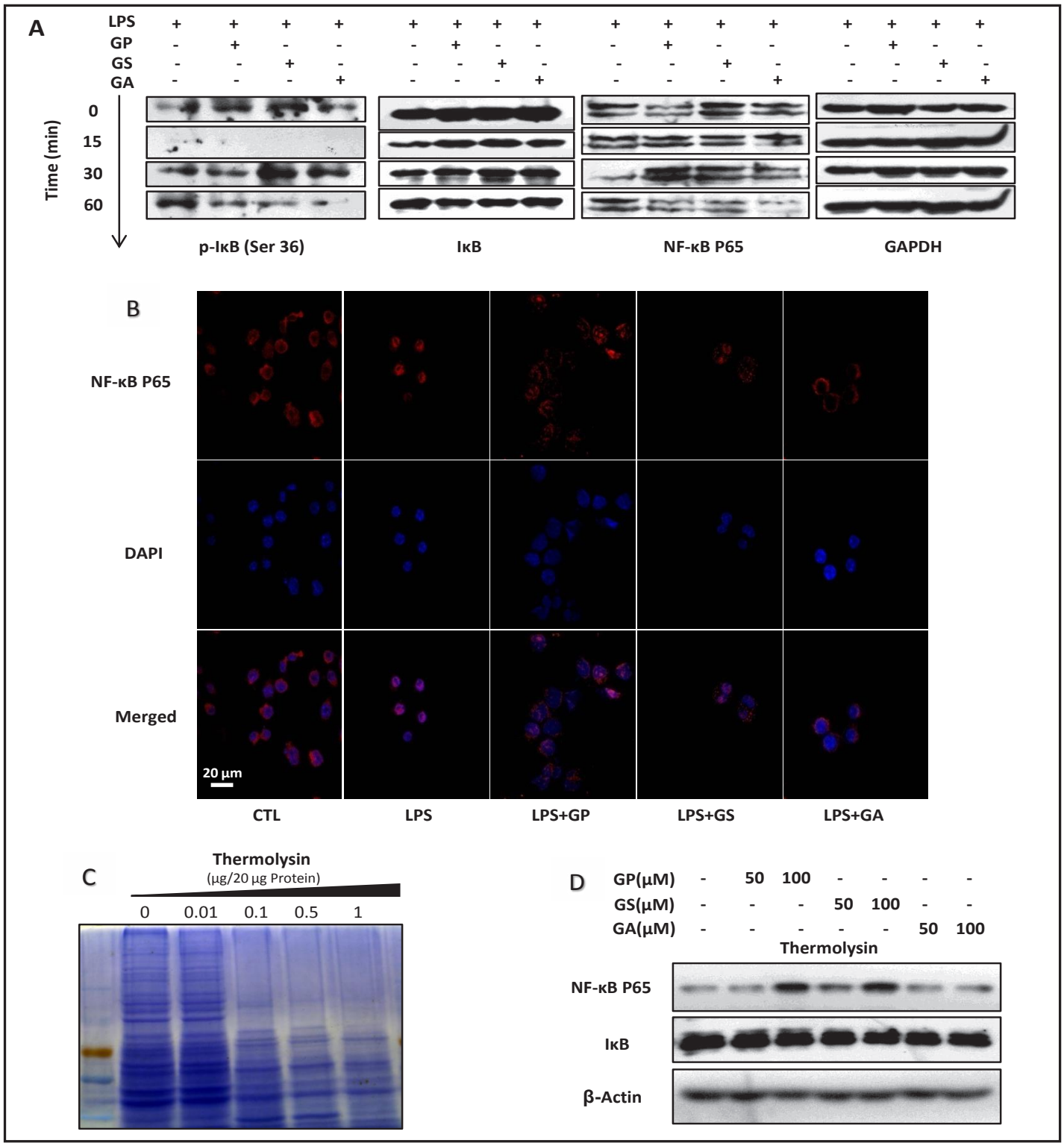

Fig. 2. RAW 264.7 cells were pre-incubated with control solution, genipin (GP, $100 \mu \mathrm{M}$ ), geniposide (GS, $100 \mu \mathrm{M})$, and geniposidic acid (GA, $100 \mu \mathrm{M})$ for 2 hours respectively, and then incubated with LPS $(2 \mu \mathrm{g} / \mathrm{mL})$ for different lengths of time $(0,15,30$, and $60 \mathrm{~min})$. The results showed that the elevated phosphorylation level, decreased expression level of I $\mathrm{B}$, and decreased NF- $\kappa \mathrm{B}$ P65 induced by LPS were all efficiently attenuated by the iridoids (A). In addition, LPS-induced nuclear transfer of NF- $\kappa$ B P65 from the cytoplasm in RAW 264.7 was also blocked by the iridoids (B). RAW 264.7 cells were treated with the iridoids of various concentrations for 30 minutes, and lysates were subjected to thermolysin digestion. Though thermolysin efficiently degraded all the proteins (C), pretreatment with genipin or geniposide could block the degradation of NF- $\kappa$ B P65 (D).

\section{KARGER}


The NF- $\kappa$ B intracellular signaling system is known as a dominant paradigm for specific signal transduction molecules, regulatory proteins and gene activation in response to inflammation [32]. It is also regarded as the core regulating factor in the process of inhibiting LPS-induced inflammation by the iridoids. In the pathogenesis of inflammation induced by LPS, IкB protein would be phosphorylated and degraded immediately after receiving the upstream signals. Next, NF- $\mathrm{BB}$ P65 would be released

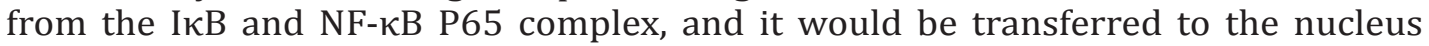
to activate the expression of inflammatory factors. In this study, the iridoids were demonstrated to have the ability to block the process efficiently and protect NF- $\kappa \mathrm{B}$ P65 from thermolysin degradation, indicating that NF- $\kappa$ B P65 was the direct target of the iridoids.

\section{Free radical scavenging ability of the iridoids}

The iridoids were utilized to react with DPPH at different rates $(0,0.25,0.5,1$ $\mathrm{mol} / \mathrm{g}$ ), but most DPPH remained (Fig. 3A, B, C). Genipin showed a slight ability to scavenge ultra oxygen free radicals, but geniposide and geniposidic acid did not (Fig.

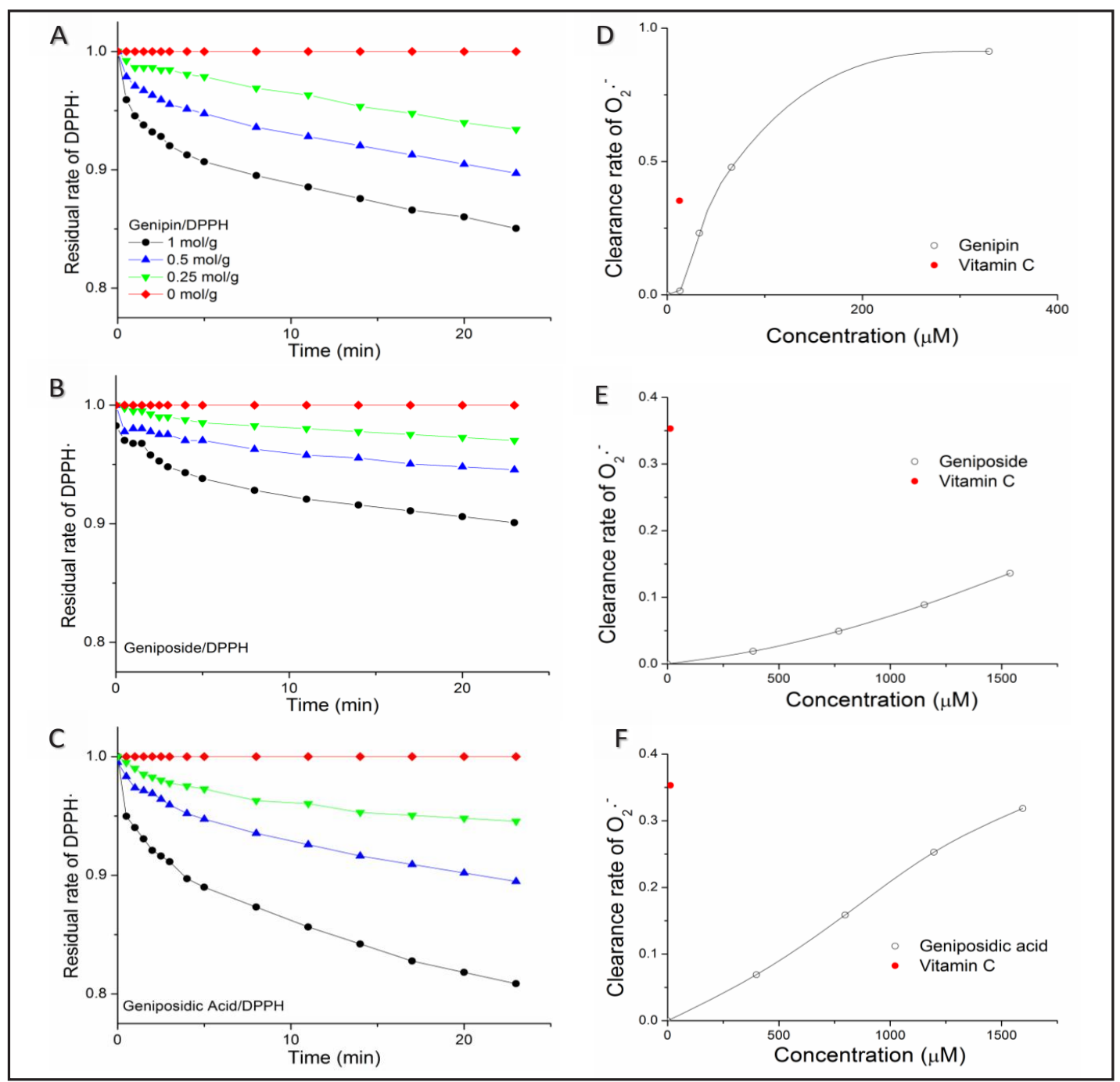

Fig. 3. Iridoids were utilized to react with DPPH at different rates $(0,0.25,0.5,1 \mathrm{~mol} / \mathrm{g})$, but most DPPH remained (A, B, C). Genipin showed slight ability to scavenge ultra oxygen free radicals, but geniposide and geniposidic acid did not (D, E, F).

\section{KARGER}




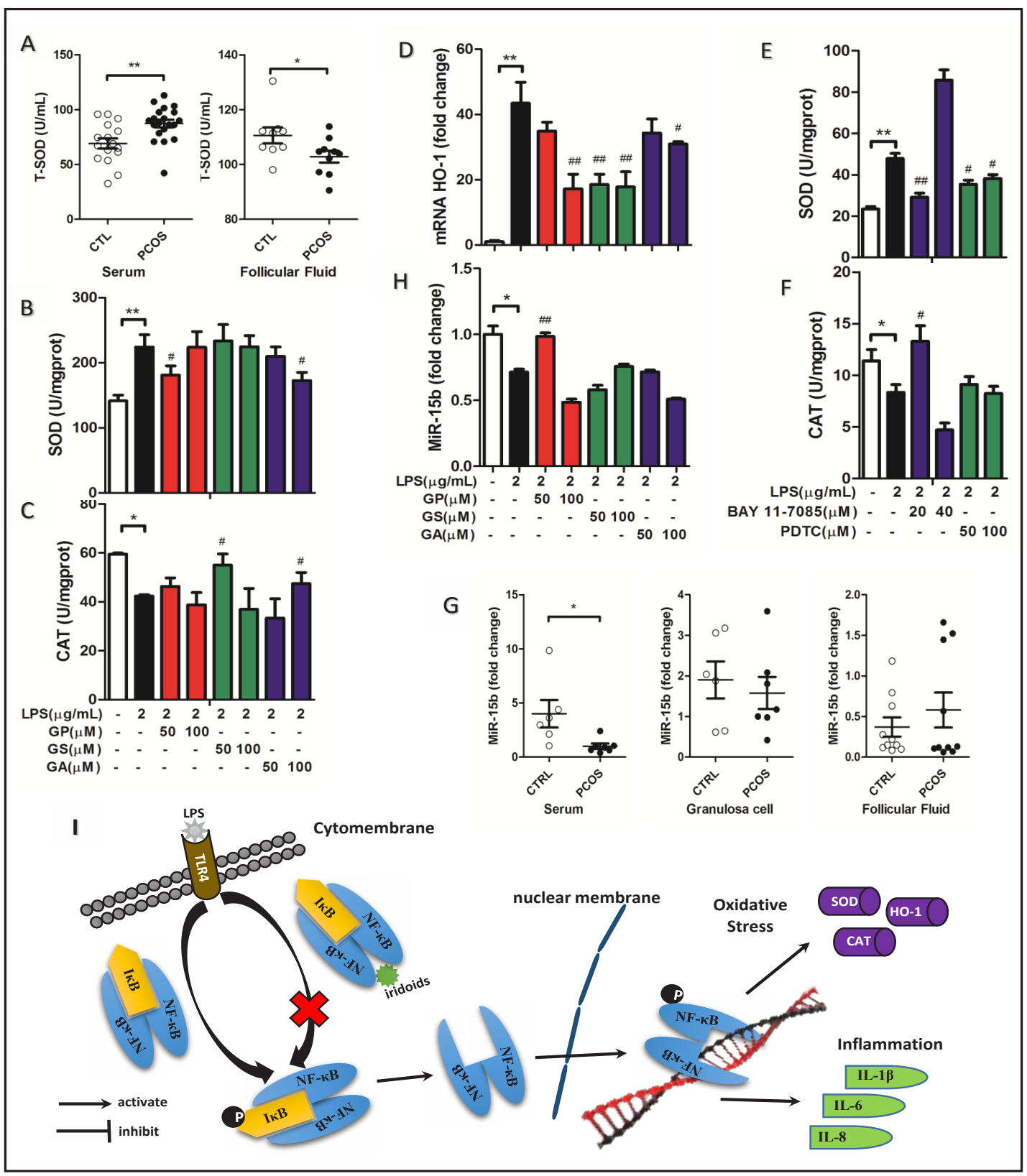

Fig. 4. Total SOD (T-SOD) activity was discovered to be altered significantly in both serum and follicular fluid of PCOS (A). Cells were incubated with control solution, LPS, and LPS plus the iridoids (genipin (GP), geniposide (GS), or geinposidic acid (GA)) of different concentrations for 24 hours. Elevated SOD activity and HO-1 mRNA expression, and reduced CAT activity induced by LPS were inhibited by the iridoids respectively in RAW 264.7 (B, C, D), as well as BAY 11-7085 and PDTC (E, F). MiR-15b expression was found to be reduced in serum of PCOS, but not in granulosa cell or follicular fluid (G). LPS efficiently inhibited the expression of MiR-15b in RAW 264.7 cells (H).The iridoids inhibited LPS-induced inflammation and oxidative stress by blocking NF- $\kappa B$ (I). ${ }^{*} \mathrm{P}<0.0 .5,{ }^{* *} \mathrm{P}<0.01$; by contrast with LPS group, ${ }^{\#} \mathrm{P}<0.05$, ${ }^{\# \#} \mathrm{P}<0.01$.

3D, E, F). In contrast with anthocyanin (an efficient cleaner of DPPH free radicals) [33] and Vitamin C (an efficient cleaner of ultra oxygen free radical), the abilities of the iridoids to scavenge free radicals could be ignored, indicating that the iridoids did not have efficient anti-oxidant abilities. 


\section{Cellular Physiology Cell Physiol Biochem 2017;43:1855-1865 and Biochemistry \begin{tabular}{l|l} 
DOI: 10.1159/000484074 \\
Published onnine: October 19, 2017
\end{tabular} $\begin{aligned} & \text { O } 2017 \text { The Author(s). Published by S. Karger AG, Basel } \\
& \text { www.karger.com/cpb }\end{aligned}$ \\ Zuo et al.: Iridoids Inhibit Inflammation and Oxidative Stress by NF- $\mathrm{KB}$}

Anti-oxidant ability of the iridoids in PCOS

Total SOD (T-SOD) activity was discovered to be altered significantly in both the serum and follicular fluid of PCOS (Fig. 4A), but GSH-Px activity and MDA level were not (see online suppl. material, Suppl. Fig. 1C). Cells were incubated with control solution, LPS, and LPS plus the iridoids of different concentrations for 24 hours. LPSinduced elevated SOD activity, increased HO-1 mRNA expression, and reduced CAT activity were inhibited by the iridoids in RAW 264.7 (Fig. 4B, C, D). BAY 11-7085 and PDTC also showed the same effect (Fig. 4E, F). However, LPS could not significantly stimulate KGN into an altered oxidative state, as none of the SOD or CAT activities changed significantly (see online suppl. material, Suppl. Fig. 1D).

SOD, CAT, GSH-Px, MDA, and HO-1 are common markers to evaluate oxidative stress levels. SOD is the enzyme to transform the superoxides into harmless chemicals and hydrogen peroxide $\left(\mathrm{H}_{2} \mathrm{O}_{2}\right)$, and CAT is the enzyme to resolve $\mathrm{H}_{2} \mathrm{O}_{2}$. GSH-Px is the enzyme to transform superoxides and promote $\mathrm{H}_{2} \mathrm{O}_{2}$ catabolism. MDA is the product of lipid oxidation. Though altered T-SOD activity was observed in both the serum and follicular fluid of PCOS, the results were completely opposite, which indicates the potential differences of oxidative stress between the systemic cycle and the regions. HO-1 was also regarded as a useful marker for oxidative stress in recent years because it was thought to play important roles against oxidative stress [28]. Though the iridoids effectively alleviated the elevated oxidative stress induced by LPS

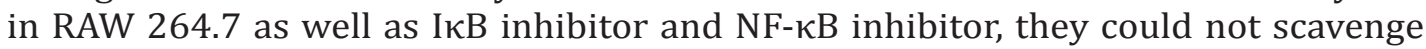
free radicals efficiently, indicating that the anti-oxidative ability of the iridoids may be derived from their anti-inflammatory ability. In addition, KGN cells did not show significant altered oxidative stress after LPS stimulation, and this phenomenon may result from its much less intense inflammatory response.

\section{Altered microRNA expression in PCOS}

MiR-15b expression was observed to be reduced in serum in PCOS but not in granulosa cells or follicular fluid (Fig. 4G). LPS efficiently inhibits the expression of MiR-15b in RAW 264.7 cells (Fig. 4H), and it also inhibits the expression of MiR-223 and MiR-93 (see online suppl. material, Suppl. Fig. 1E). Low dosage genipin could enhance the reduced MiR-15b expression. The roles of various microRNAs played in PCOS have attracted much attention in recent years. MiR-15b has been demonstrated to regulate cell proliferation and apoptosis, which are associated with some characteristics of PCOS. LPS-induced MiR-15b expression was consistent with that of PCOS, indicating that chronic inflammation contributes to the abnormal miRNA expression of PCOS. In addition, LPS could also inhibit the expression of MiR-223 and MiR-93, which were related to adipogenic differentiation and insulin resistance, respectively.

\section{Conclusion}

Geniposidic acid, an iridoid of genipin stem nucleus, and genipin and geniposide, were demonstrated toinhibit the inflammatory response efficiently. NF- $\kappa B$ was thought to be the target of the iridoids, and blocking the NF- $\kappa B$ signaling pathway was the mechanism of inhibiting inflammation. Chronic inflammation was an incentive of oxidative stress in PCOS, and the inflammation-induced oxidative stress status may be variable, depending on different conditions. The iridoids exhibited a low ability to interfere with oxidative stress, but their efficient anti-inflammatory ability provided a pathway to attenuate oxidative stress. (Fig. 4I) Chronic inflammation also seemed to be related to the altered miRNA expression in PCOS, which was not strongly affected by iridoid treatment. 


\section{Cellular Physiology Cell Physiol Biochem 2017;43:1855-1865 \begin{tabular}{ll|l} 
and Biochemistry Published onlIne: October 19, 2017 & $\begin{array}{l}\text { (c) } 2017 \text { The Author(s). Published by S. Karger AG, Basel } \\
\text { www.karger.com/cpb }\end{array}$ \\
\hline
\end{tabular}}

\section{Acknowledgements}

The study was supported by the Science and Technology Department of Chengdu under 2014-HM01-00247-SF. Sincere thanks are due to Dr. Ma Yangxian for providing the RAW 264.7 cell line and to Dr. Wang Fei for the KGN cell line. Thanks are also due to Li Xiaoliang for providing helpful assistance. All of the authors declared that there was no interest of conflict.

\section{Disclosure Statemen}

The contents of our manuscript are all from research of all authors and do not involve any commercial and national secrets.

\section{References}

1 Zuo T, Zhu M, Xu W: Roles of Oxidative Stress in Polycystic Ovary Syndrome and Cancers. Oxid Med Cell Longev 2016;1:1-14.

2 Zhao Y, Zhang C, Huang Y, Yu Y, Li R, Li M, Liu N, Liu P, Qiao J: Up-regulated expression of WNT5a increases inflammation and oxidative stress via PI3K/AKT/NF-kappaB signaling in the granulosa cells of PCOS patients. J Clin Endocrinol Metab 2015;100:201-211.

- Cui N, Wang H, Wang W, Zhang J, Xu Y, Jiang L, Yang A, Hao G: Impact of Body Mass Index on Outcomes of In vitro Fertilization/Intracytoplasmic Sperm Injection Among Polycystic Ovarian Syndrome Patients. Cell Physiol Biochem 2016;39:1723-1734.

-4 Pabalan N, Montagna E, Singian E, Tabangay L, Jarjanazi H, Barbosa CP, Bianco B: Associations of Polymorphisms in Anti-Mullerian Hormone (AMH Ile49Ser) and its Type II Receptor (AMHRII -482 A>G) on Reproductive Outcomes and Polycystic Ovary Syndrome: a Systematic Review and Meta-Analysis. Cell Physiol Biochem 2016;39:2249-2261.

5 Duleba AJ, Dokras A: Is PCOS an inflammatory process? Fertil Steril 2012;97:7-12.

6 Sorensen AE, Wissing ML, Salo S, Englund AL, Dalgaard LT: MicroRNAs Related to Polycystic Ovary Syndrome (PCOS). Genes (Basel) 2014;5:684-708.

7 Singh RP, Massachi I, Manickavel S, Singh S, Rao NP, Hasan S, Mc Curdy DK, Sharma S, Wong D, Hahn BH, Rehimi H: The role of miRNA in inflammation and autoimmunity. Autoimmun Rev 2013;12:1160-1165.

-8 Ding CF, Chen WQ, Zhu YT, Bo YL, Hu HM, Zheng RH: Circulating microRNAs in patients with polycystic ovary syndrome. Hum Fertil (Camb) 2015;18:22-29.

-9 Zhao C, Liu X, Shi Z, Zhang J, Zhang J, Jia X, Ling X: Role of serum miRNAs in the prediction of ovarian hyperstimulation syndrome in polycystic ovarian syndrome patients. Cell Physiol Biochem 2015;35:10861094.

10 He X, Wang J, Li M, Hao D, Yang Y, Zhang C, He R, Tao R: Eucommia ulmoides Oliv.: ethnopharmacology, phytochemistry and pharmacology of an important traditional Chinese medicine. J Ethnopharmacol 2014;151:78-92.

11 Li Y, Han C, Wang J, Xiao W, Wang Z, Zhang J, Yang Y, Zhang S, Ai C: Investigation into the mechanism of Eucommia ulmoides Oliv. based on a systems pharmacology approach. J Ethnopharmacol 2014;151:452460.

12 Zuo T, Xu W, Li H, Song H, Zhu M: Geniposide and geniposidic acid, modified forms of genipin, attenuate genipin-induced mitochondrial apoptosis without altering the anti-inflammatory ability in KGN cell line. Med Chem Res 2017;26:499-508.

13 Araki R, Hiraki Y, Yabe T: Genipin attenuates lipopolysaccharide-induced persistent changes of emotional behaviors and neural activation in the hypothalamic paraventricular nucleus and the central amygdala nucleus. Eur J Pharmacol 2014;741:1-7.

14 Cho HI, Kim SJ, Choi JW, Lee SM: Genipin alleviates sepsis-induced liver injury by restoring autophagy. Br J Pharmacol 2016;173:980-991. 


\section{Cellular Physiology Cell Physiol Biochem 2017;43:1855-1865

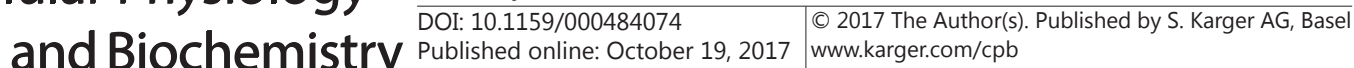

15 Kim JS, Kim SJ, Lee SM: Genipin attenuates sepsis-induced immunosuppression through inhibition of T lymphocyte apoptosis. Int Immunopharmacol 2015;27:15-23.

16 Lee CH, Kwak SC, Kim JY, Oh HM, Rho MC, Yoon KH, Yoo WH, Lee MS, Oh J: Genipin inhibits RANKL-induced osteoclast differentiation through proteasome-mediated degradation of c-Fos protein and suppression of NF-kappaB activation. J Pharmacol Sci 2014;124:344-353.

17 Kim TH, Yoon SJ, Lee SM: Genipin attenuates sepsis by inhibiting Toll-like receptor signaling. Mol Med 2012;18:455-465.

18 Rajanbabu V, Galam L, Fukumoto J, Enciso J, Tadikonda P, Lane TN, Bandyopadhyay S, Parthasarathy PT, Cho Y, Cho SH, Lee YC, Lockey RF, Kolliputi N: Genipin suppresses NLRP3 inflammasome activation through uncoupling protein-2. Cell Immunol 2015;297:40-45.

19 Wang Qiangsong, Xiang Yaozu, Cui Yuan-Lu, Lin Ke-Ming, Xin-Fang Z: Dietary Blue Pigments Derived from Genipin, Attenuate Inflammation by Inhibiting LPS-Induced iNOS and COX-2 Expression via the NF-kB Inactivation. PLOS ONE 2012;7:e34122.

20 Li CC, Hsiang CY, Lo HY, Pai FT, Wu SL, Ho TY: Genipin inhibits lipopolysaccharide-induced acute systemic inflammation in mice as evidenced by nuclear factor-kappaB bioluminescent imaging-guided transcriptomic analysis. Food Chem Toxicol 2012;50:2978-2986.

21 Liu J, Yin F, Zheng X, Jing J, Hu Y: Geniposide, a novel agonist for GLP-1 receptor, prevents PC12 cells from oxidative damage via MAP kinase pathway. Neurochem Int 2007;51:361-369.

-22 Ma TT, Li XF, Li WX, Yang Y, Huang C, Meng XM, Zhang L, Li J: Geniposide alleviates inflammation by suppressing MeCP2 in mice with carbon tetrachloride-induced acute liver injury and LPS-treated THP-1 cells. Int Immunopharmacol 2015;29:739-747.

-23 Deng Y, Guan M, Xie X, Yang X, Xiang H, Li H, Zou L, Wei J, Wang D, Deng X: Geniposide inhibits airway inflammation and hyperresponsiveness in a mouse model of asthma. Int Immunopharmacol 2013;17:561567.

24 Li F, Li W, Li X, Li F, Zhang L, Wang B, Huang G, Guo X, Wan L, Liu Y, Zhang S, Kang S, Ma J: Geniposide attenuates inflammatory response by suppressing P2Y14 receptor and downstream ERK1/2 signaling pathway in oxygen and glucose deprivation-induced brain microvascular endothelial cells. J Ethnopharmacol 2016;185:77-86.

25 Shi Q Cao J, Fang L, Zhao H, Liu Z, Ran J, Zheng X, Li X, Zhou Y, Ge D, Zhang H, Wang L, Ran Y, Fu J: Geniposide suppresses LPS-induced nitric oxide, PGE2 and inflammatory cytokine by downregulating NFkappaB, MAPK and AP-1 signaling pathways in macrophages. Int Immunopharmacol 2014;20:298-306.

-26 Song X, Zhang W, Wang T, Jiang H, Zhang Z, Fu Y, Yang Z, Cao Y, Zhang N: Geniposide plays an antiinflammatory role via regulating TLR4 and downstream signaling pathways in lipopolysaccharide-induced mastitis in mice. Inflammation 2014;37:1588-1598.

27 Wang J, Hou J, Zhang P, Li D, Zhang C, Liu J: Geniposide reduces inflammatory responses of oxygen-glucose deprived rat microglial cells via inhibition of the TLR4 signaling pathway. Neurochem Res 2012;37:22352248.

-28 Yin F, Liu J, Zheng X, Guo L, Xiao H: Geniposide induces the expression of heme oxygenase-1 via PI3K/ Nrf2-signaling to enhance the antioxidant capacity in primary hippocampal neurons. Biol Pharm Bull 2010;33:1841-1846.

29 Guan L, Feng H, Gong D, Zhao X, Cai L, Wu Q Yuan B, Yang M, Zhao J, Zou Y: Genipin ameliorates age-related insulin resistance through inhibiting hepatic oxidative stress and mitochondrial dysfunction. Exp Gerontol 2013;48:1387-1394.

-30 Koriyama Y, Chiba K, Yamazaki M, Suzuki H, Muramoto K, Kato S: Long-acting genipin derivative protects retinal ganglion cells from oxidative stress models in vitro and in vivo through the Nrf2/antioxidant response element signaling pathway. J Neurochem 2010;115:79-91.

-31 Lomenick B, Hao R, Jonai N, Chin RM, Aghajan M, Warburton S, Wang J, Wu RP, Gomez F, Loo JA, Wohlschlegel JA, Vondriska TM, Pelletier J, Herschman HR, Clardy J, Clarke CF, Huang J: Target identification using drug affinity responsive target stability (DARTS). Curr Protoc Chem Biol 2009;3:163-180.

-32 Ivanenkov YA, Balakin KV, Lavrovsky Y: Small molecule inhibitors of NF-kB and JAK/STAT signal transduction pathways as promising anti-inflammatory therapeutics. Mini Rev Med Chem 2011;11:55-78.

-33 Wang LJ, Su S, Wu J, Du H, Li SS, Huo JW, Zhang Y, Wang LS: Variation of anthocyanins and flavonols in Vaccinium uliginosum berry in Lesser Khingan Mountains and its antioxidant activity. Food Chem 2014;160:357-364. 